\title{
Pegylated interferon- $\alpha$ protects type 1 pneumocytes against SARS coronavirus infection in macaques
}

\author{
Bart L Haagmans ${ }^{1,6}$, Thijs Kuiken ${ }^{1,6}$, Byron E Martina ${ }^{1}$, Ron A M Fouchier ${ }^{1}$, Guus F Rimmelzwaan ${ }^{1}$, \\ Geert van Amerongen ${ }^{1}$, Debby van Riel $^{2}$, Ton de Jong ${ }^{3}$, Shigeyuki Itamura ${ }^{4}$, Kwok-Hung Chan ${ }^{5}$, \\ Masato Tashiro $^{4}$ \& Albert D M E Osterhaus ${ }^{1}$
}

The primary cause of severe acute respiratory syndrome (SARS) is a newly discovered coronavirus ${ }^{1-7}$. Replication of this SARS coronavirus (SCV) occurs mainly in the lower respiratory tract, and causes diffuse alveolar damage ${ }^{2,7,8}$. Lack of understanding of the pathogenesis of SARS has prevented the rational development of a therapy against this disease. Here we show extensive SCV antigen expression in type 1 pneumocytes of experimentally infected cynomolgus macaques (Macaca fascicularis) at $4 \mathrm{~d}$ postinfection (d.p.i.), indicating that this cell type is the primary target for SCV infection early in the disease, and explaining the subsequent pulmonary damage. We also show that prophylactic treatment of SCVinfected macaques with the antiviral agent pegylated interferon- $\alpha($ IFN- $\alpha)$ significantly reduces viral replication and excretion, viral antigen expression by type 1 pneumocytes and pulmonary damage, compared with untreated macaques. Postexposure treatment with pegylated IFN- $\alpha$ yielded intermediate results. We therefore suggest that pegylated IFN- $\alpha$ protects type 1 pneumocytes from SCV infection, and should be considered a candidate drug for SARS therapy

SARS has recently emerged in the human population as a potentially fatal respiratory disease. Severely affected patients develop acute respiratory distress syndrome ${ }^{7-10}$, which corresponds with diffuse alveolar damage (DAD) at autopsy ${ }^{2,11}$. A newly discovered coronavirus, SCV, has been identified as the primary cause of SARS ${ }^{1,2,5-7}$. SARS patients have been treated empirically with a combination of ribavirin, oseltamivir, antibiotics and corticosteroids ${ }^{7-9}$. However, neither case-control studies nor animal models have been used to determine the efficacy of these drugs. The choice and evaluation of potential drugs is also hampered by poor understanding of the pathogenesis of SARS.

We previously hypothesized that SCV infects type 1 pneumocytes, based on the character of the pulmonary lesions in experimentally infected macaques and the known tropism of other respiratory coronaviruses for type 1 pneumocytes ${ }^{2}$. We found extensive type 2 pneumo- cyte hyperplasia in the lungs of SCV-infected macaques at 6 d.p.i., suggesting that there had been loss of type 1 pneumocytes at an earlier stage of infection ${ }^{2,12}$. To determine whether type 1 pneumocytes are infected by SCV at an earlier stage of infection, we conducted pathological and immunohistochemical examinations of the lungs of four SCV-infected macaques (control group) at 4 d.p.i.. The lungs had multifocal, acute $\mathrm{DAD}$, which was characterized by flooding of alveoli with protein-rich edema fluid mixed with neutrophils and rare syncytia, extensive loss of alveolar and bronchiolar epithelium, and occasional type 2 pneumocyte hyperplasia (Fig. 1a; for comparison, normal alveolar morphology is seen in Fig. 1b). Immunohistochemistry revealed extensive SCV antigen expression by squamous cells lining the alveolar walls (Fig. 1c,d; compare with Fig. 1e). These cells were identified as type 1 pneumocytes by their location, morphology and expression of keratin in serial sections. Transmission electron microscopy of alveolar cells revealed coronaviruslike particles measuring about $70 \mathrm{~nm}$ in diameter with typical internal nucleocapsid-like structure (Fig. 1f,g). These cells were identified as type 1 pneumocytes because they lined the alveolar lumen, were closely apposed to the basement membrane, were squamous, contained abundant pinocytotic vesicles, and-in contrast to type 2 pneumocytes - had neither lamellar bodies nor microvilli ${ }^{13}$. As found previously in experimentally infected macaques at 6 d.p.i., less extensive SCV antigen expression also was detected in hyperplastic type 2 pneumocytes within inflammatory foci ${ }^{2}$ (Fig. 1h). Together, these histopathologic and immunohistochemical findings show that type 1 pneumocytes are the main target of SCV in early infection, and are associated with DAD.

An effective antiviral agent for the treatment of SCV infection is needed because decreasing viral replication in the first phase of SARS may reduce the severity of subsequent pathological damage, as has been found in another model of viral-induced $\mathrm{DAD}^{14,15}$. IFN- $\alpha$ may inhibit replication of animal and human coronaviruses ${ }^{16-18}$. As a candidate drug, we tested the efficacy of pegylated recombinant IFN- $\alpha 2 b$, a registered drug for the treatment of chronic hepatitis $\mathrm{C}^{19}$. We chose this drug because it is registered and therefore directly available, because human recombinant IFNs inhibit SCV replication in vitro ${ }^{20}$, and because pegylation optimizes the

${ }^{1}$ Department of Virology, ${ }^{2}$ Department of Immunology and ${ }^{3}$ Department of Pathology, Erasmus Medical Centre, P0 Box 1738,3000 DR, Rotterdam, Netherlands ${ }^{4}$ Department of Viral Diseases \& Vaccine Control, National Institute of Infectious Diseases, Tokyo 208-0011, Japan. ${ }^{5}$ Department of Microbiology and Pathology, Queen Mary Hospital, University of Hong Kong SAR, China. ${ }^{6}$ These authors contributed equally to this work. Correspondence should be addressed to A.D.M.E.O. (a.osterhaus@erasmusmc.nl).

Published online 22 February 2004; doi:10.1038/nm1001 
a

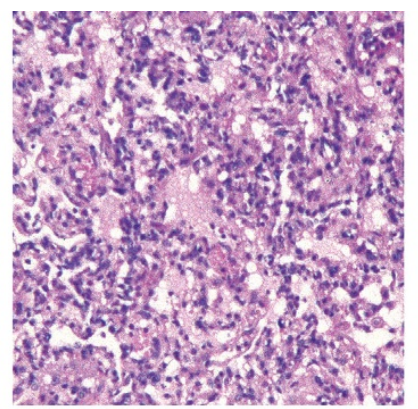

C

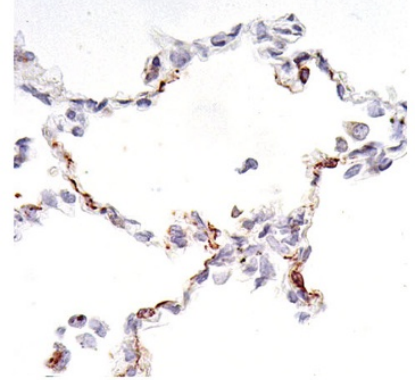

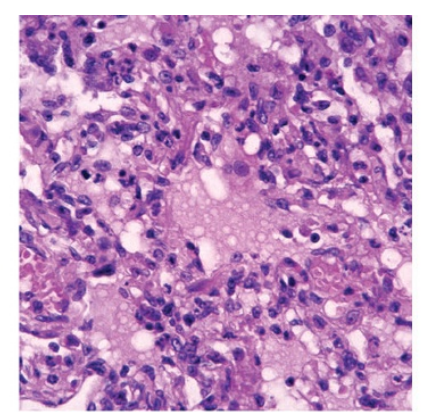

d

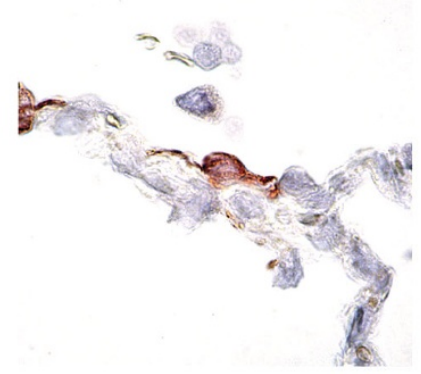

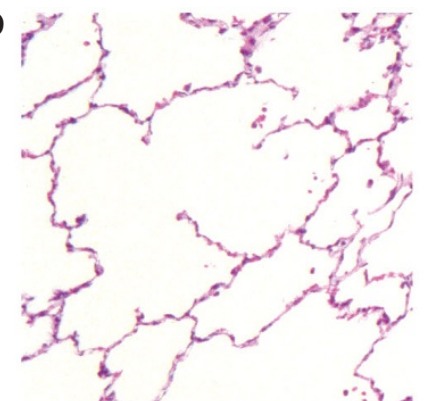

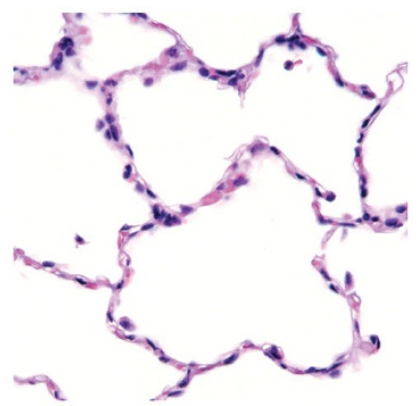

e
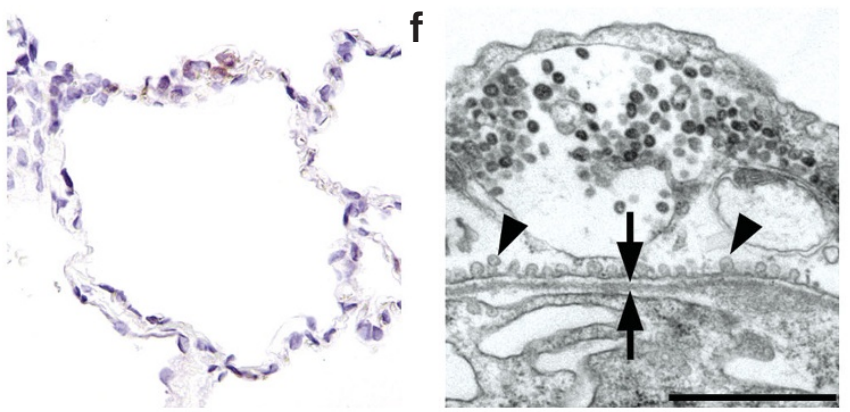

Figure 1 Histological lesions and immunohistochemical and ultrastructural detection of SCV in lungs of experimentally infected macaques. (a) DAD in pulmonary alveoli (control group). (b) Normal pulmonary alveoli (prophylactic group). (c,d) SCV antigen expression in type 1 pneumocytes (control group).

(e) Lack of SCV antigen expression in pulmonary alveoli (prophylactic group).

(f) Viral nucleocapsids in cytoplasmic vesicle of type 1 pneumocyte (control group), showing pinocytotic vesicles (arrowheads) and basement membrane (arrows). Scale bar, $1 \mu \mathrm{m}$. (g) Higher magnification of viral nucleocapsids shown in f. Scale bar, $200 \mathrm{~nm}$. (h) SCV antigen expression in type 2 pneumocytes (control group). Sections were stained with $\mathrm{H} \& \mathrm{E}(\mathbf{a}, \mathbf{b})$, for SCV antigen (c-e,h), or with uranyl acetate and lead citrate $(\mathbf{f}, \mathbf{g})$. Original magnification, $\times 25$ (a left, b left), $\times 100$ (a right, b right, $\mathbf{c}, \mathbf{e}$ ) or $\times 250$ (d,h).

g

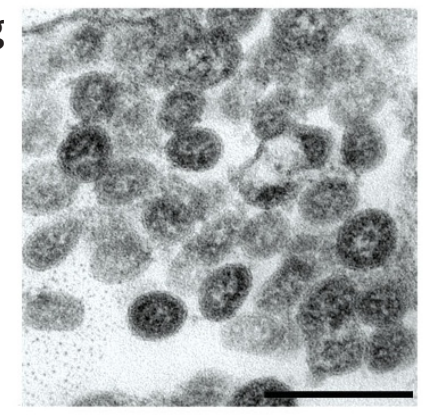

h

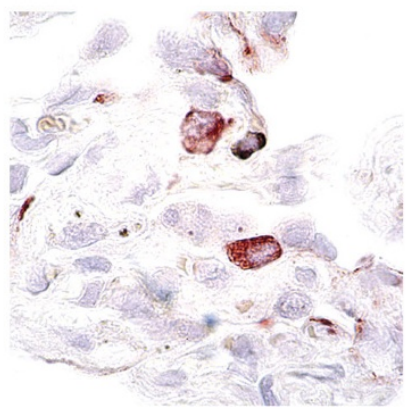

pharmacokinetic properties of IFN ${ }^{21}$. We first tested the effect of pegylated IFN- $\alpha$ on SCV-infected Vero cells, and observed a dose-dependent antiviral effect (Fig. 2a). We then determined the plasma levels of pegylated IFN- $\alpha$ after intramuscular injection into a group of six macaques (prophylactic group). High plasma levels were attained $1 \mathrm{~d}$ after injection (Fig. 2b), similar to peak levels found in patients after subcutaneous injection with $3-\mu \mathrm{g} / \mathrm{kg}$ pegylated IFN- $\alpha^{21}$. Because IFN- $\alpha$ is known to activate macrophages ${ }^{22}$, we measured plasma levels of neopterin after pegylated IFN- $\alpha$ treatment, as a measure of macrophage activation. Neopterin levels were increased in all animals (Fig. 2c), confirming the biological availability of pegylated IFN- $\alpha$ in the treated macaques.

To evaluate the prophylactic use of pegylated IFN- $\alpha$, we experimentally infected the macaques in the prophylactic group with SCV $3 \mathrm{~d}$ after the start of pegylated IFN- $\alpha$ treatment, and compared virological and

pathological parameters with a control group of four macaques treated with PBS. We limited our investigation to pharyngeal swabs and the lung because an earlier study did not provide evidence of extensive viral replication in other organs ${ }^{2}$.We found that all parameters were significantly $(P<0.05)$ reduced in the prophylactic group compared with the control group. Virus excretion from the pharynx was abrogated (Fig. 3), and the virus titer in the lungs at 4 d.p.i. was significantly $(P<0.01)$ reduced (Fig. 4a). Immunohistochemistry showed that expression of SCV antigen in type 1 pneumocytes was reduced by $90 \%$ (Figs. 1e and 4b). By pathology, the extent and severity of DAD was reduced by $80 \%$ (Figs. $1 \mathrm{~b}$ and 4c). These data indicate that prophylactic use of pegylated IFN- $\alpha$ substantially, although not completely, protects type 1 pneumocytes of experimentally infected macaques from SCV infection, with abrogation of virus excretion and reduced severity of pulmonary lesions.

a

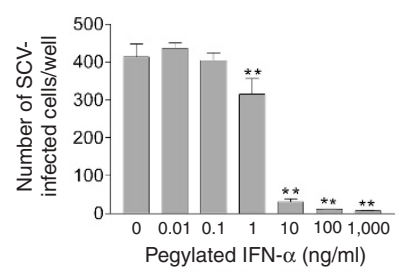

b

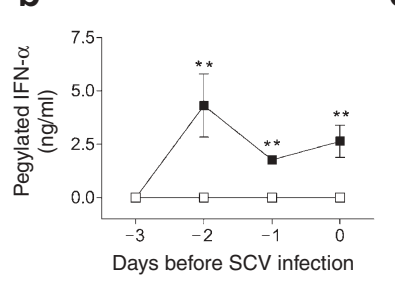

C

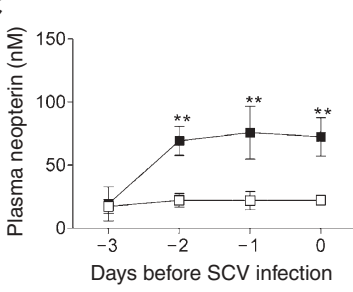

Figure 2 Antiviral activity of pegylated IFN- $\alpha$ against SCV in vitro and its biological activity in macaques. (a) Effect of pegylated IFN- $\alpha$ against SCV infection in vitro. Similar results were obtained in three separate experiments. (b) Pharmacokinetic analysis of pegylated IFN- $\alpha(\mathbf{b})$ and induction of neopterin (c) in macaques treated with PBS ( $\square$, control group; $n=4)$ or pegylated IFN- $\alpha(\mathbf{\square}$, prophylactic group; $n=4$ ) on days -3 and -1 . Data are expressed as mean \pm s.d. ${ }^{* *}, P<0.01$ compared with control. 


\section{LET TER S}

To test the efficacy of pegylated IFN- $\alpha$ as an antiviral agent postexposure, we injected pegylated IFN- $\alpha$ intramuscularly into a postexposure group of four macaques, 1 and $3 \mathrm{~d}$ after experimental SCV infection, and evaluated them in the same way as the prophylactic group. Excretion of SCV from the pharynx was found only 2 d.p.i., at a significantly $(P<0.05)$ reduced level compared with the control group (Fig. 3). Moreover, the virus titer in the lungs at 4 d.p.i. was significantly $(P<0.01)$ decreased, whereas the remaining parameters were less reduced (Fig. $4 \mathrm{a}-\mathrm{c}$ ). These results show that use of pegylated IFN- $\alpha 1 \mathrm{~d}$ postexposure protects type 1 pneumocytes of experimentally infected macaques from SCV infection, but is less effective than prophylactic use.

In this study, we have shown that type 1 pneumocytes are the main target cells for SCV infection of cynomolgus macaques early in the disease, and that pegylated IFN- $\alpha$ protects type 1 pneumocytes from SCV infection. The first point-type 1 pneumocytes as the primary target cell-is evident from the extensive presence of SCV in type 1 pneumocytes at 4 d.p.i. (Fig. 1c,d). Targeting of type 1 pneumocytes by SCV explains why clinical disease and lesions are centered on the lower respiratory tract ${ }^{7,11,23}$, and fits with the lesions in early fatal SARS cases in humans $s^{11,23}$ and in experimentally infected macaques euthanized at 6 d.p.i. (ref. 2). When the pathological studies in humans and macaques are examined together, the following temporal sequence of lung lesions emerges: viral infection and subsequent loss of type 1 pneumocytes; acute DAD characterized by flooding of alveolar lumina with highly proteinaceous edema fluid; chronic DAD characterized by type 2 pneumocyte hyperplasia; and, in severe cases, extensive pulmonary fibrosis. This sequence of events corresponds to the stereotypic alveolar reaction to acute lung injury from a variety of causes ${ }^{12}$. More detailed experimental studies will be needed to identify other possible target cells and further elucidate the pathogenesis of SARS.

The second point - that pegylated IFN- $\alpha$ protects type 1 pneumocytes from SCV infection-is based on the beneficial effect of pegylated IFN- $\alpha$ therapy initiated $3 \mathrm{~d}$ before SCV inoculation of macaques. In these macaques, SCV infection of type 1 pneumocytes and severity of lung lesions were significantly reduced (Fig. 4), and viral excretion was abrogated (Fig. 3). Pegylated IFN- $\alpha$ treatment may have an important influence on the outcome of SARS. In both virusinduced $^{14,15}$ and toxin-induced ${ }^{24} \mathrm{DAD}$, the severity of pulmonary damage is dose dependent, with higher doses causing increased pulmonary fibrosis, loss of lung function and mortality. Reduction of the viral load by pegylated IFN- $\alpha$ therapy at an early stage of SCV infection might therefore help prevent serious or fatal outcome of SARS

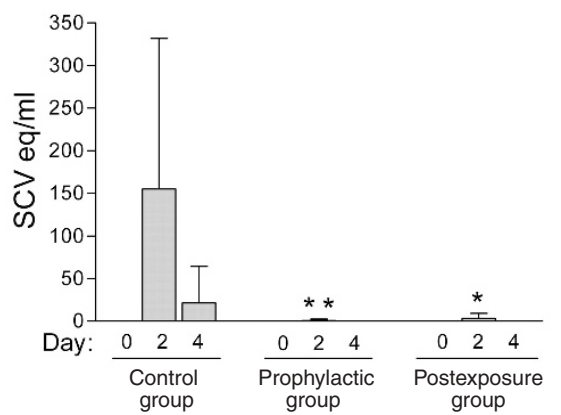

Figure 3 Effect of pegylated IFN- $\alpha$ on SCV excretion in macaques. SCV detection in pharyngeal swabs taken at 0,2 or 4 d.p.i. from macaques treated with PBS (control group; $n=4$ ) or pegylated IFN- $\alpha$ prophylactically $(n=6)$ or postexposure $(n=4)$. Eq, equivalents. Data are expressed as mean \pm s.d. * $P<0.05$; ${ }^{*}, P<0.01$ compared with control group at 2 d.p.i.

associated with pulmonary fibrosis. In addition to potential disease mitigation, reduced viral excretion through pegylated IFN- $\alpha$ therapy may also have an epidemiological effect by reducing the spread of SCV in the human population. Whether the mechanism of pegylated IFN- $\alpha$ protection is by direct antiviral activity or immunostimulatory effects ${ }^{25}$ remains to be determined.

The reduced protection of type 1 pneumocytes by pegylated IFN- $\alpha$ in the postexposure group compared with the prophylactic group was probably because the initiation of therapy (at 1 d.p.i.) was too close to the peak of viral infection in the lungs (at 2 d.p.i.; Fig. 3). Thus, substantial infection of type 1 pneumocytes may already have occurred by the time pegylated IFN- $\alpha$ reached therapeutic levels. These in vivo findings are in accordance with the reduced in vitro activity against SCV of IFN- $\alpha$ added after infection, compared with IFN- $\alpha$ added before infection ${ }^{20}$. It also corresponds to the results of IFN therapy against acute virus infections in most animal models, where IFN is most effective when administered before virus inoculation or before the peak of virus replication in the target organ ${ }^{26}$.

The time interval during which effective postexposure treatment with pegylated IFN- $\alpha$ can be initiated may be longer in humans than in experimentally infected macaques. This is because the peak of SCV infection in the lungs occurs around 16 d.p.i. in humans-based on an average incubation period of $6 \mathrm{~d}$ (refs. 9,27) and a peak in viral excretion $10 \mathrm{~d}$ after onset of symptoms ${ }^{28}$ — compared with 2 d.p.i. in the macaques (Fig. 3).

In conclusion, these studies show that type 1 pneumocytes are the main target cells for SCV infection of macaques early in the disease, and that pegylated IFN- $\alpha$, a commercially available antiviral drug, protects these cells from SCV infection. We believe that these preliminary results warrant clinical studies with pegylated IFN- $\alpha$, if and when SARS re-emerges. Prophylactic or early postexposure treatment with pegylated IFN- $\alpha$ may help reduce the impact of SCV infection on health-care workers and others possibly exposed to SCV, and may limit the spread of the virus in the human population.

\section{METHODS}

Antiviral assay. Wells containing Vero cells were treated in triplicate with pegylated recombinant IFN- $\alpha$ (PEG-Intron, Schering) for $16 \mathrm{~h}$ and infected 
with 100 median tissue culture infectious dose $\left(\mathrm{TCID}_{50}\right)$ /well SCV (obtained from patient 5688, who died of SARS ${ }^{2}$ ). After $16 \mathrm{~h}$, the supernatant was removed and cells were fixed with 10\% neutral-buffered formalin and 70\% ethanol (10 min at room temperature). SCV antigen-positive cells were visualized by immunohistochemistry, as described below. The numbers of SCV-infected cells per well were summarized as mean \pm s.d.

Macaque studies. Approval for animal experiments was obtained from the Institutional Animal Welfare Committee. Three groups of cynomolgus macaques were infected intratracheally with $1 \times 10^{6} \mathrm{TCID}_{50}$ SCV suspended in $5 \mathrm{ml}$ of PBS. The control group $(n=4)$ was injected intramuscularly with PBS, while the prophylactic $(n=6)$ and postexposure $(n=4)$ groups were treated with pegylated IFN- $\alpha$ at a dose of $3 \mathrm{mg} / \mathrm{kg}$. The prophylactic group was injected with pegylated IFN- $\alpha$ on days $-3,-1,1$ and 3 after SCV infection; the postexposure group was injected on days 1 and 3 after SCV infection. Four macaques from each group were euthanized on day 4 after infection. At days $-3,-2,-1,0,+2$ and +4 , we anesthetized the macaques with ketamine, collected $10 \mathrm{ml}$ blood from inguinal veins and took pharyngeal swabs, which were placed in $1 \mathrm{ml}$ transport medium ${ }^{29}$. Pharyngeal swabs were frozen at $-70^{\circ} \mathrm{C}$ until RT-PCR analysis. Pegylated IFN- $\alpha$ levels were determined using ELISA (Bender MedSystems Diagnostics) with PEG-Intron as a standard. Neopterin levels were determined as described ${ }^{22}$. Necropsies were done according to a standard protocol; one lung of each monkey was inflated with $10 \%$ neutral-buffered formalin by intrabronchial intubation and suspended in $10 \%$ neutral-buffered formalin overnight. Samples were collected in a standard manner (one from the cranial part of the lung, one from the medial part and two from the caudal part), embedded in paraffin, cut at $5 \mu \mathrm{m}$ and used for immunohistochemistry (see below) or stained with H\&E.

For semiquantitative assessment of SCV infection-associated inflammation in the lung, each H\&E-stained section was examined for inflammatory foci by light microscopy using a $10 \times$ objective. Each focus was scored for size $(1$, smaller than or equal to area of $10 \times$ objective; 2 , larger than area of $10 \times$ objective and smaller than or equal to area of $2.5 \times$ objective; 3 , larger than area of $2.5 \times$ objective) and severity of inflammation (1, mild; 2 , moderate; 3 , marked). The cumulative scores for the inflammatory foci provided the total score per animal. Sections were examined without knowledge of the identity of the macaques. The lung sections of one monkey in the postexposure group were not assessed because of the presence of inflammation from premortem aspiration of food remains. Lung samples from a control macaque were used for transmission electron microscopy as described ${ }^{2}$.

Three lung tissue samples taken from the other lung (one from the cranial part of the lung, one from the medial part and one from the caudal part) were homogenized in $2 \mathrm{ml}$ transport medium ${ }^{29}$ using Polytron PT2100 tissue grinders (Kinematica). After low-speed centrifugation, the homogenates were frozen at $-70{ }^{\circ} \mathrm{C}$ until inoculation on Vero 118 cell cultures in tenfold serial dilutions. The identity of the isolated virus was confirmed as SCV by RT-PCR of the supernatant.

Immunohistochemistry. The same formalin-fixed, paraffin-embedded lung samples that were used for histology - one from the cranial part of the lung, one from the medial part and two from the caudal part-were cut at $5 \mu \mathrm{m}$ and stained for SCV antigen using either biotinylated purified human IgG from a convalescent SARS patient, negative-control biotinylated purified human IgG, or the dilution buffer, as described previously ${ }^{2}$. Twenty-five arbitrarily chosen $20 \times$ objective fields of lung parenchyma in each lung section were examined by light microscopy for the presence of SCV antigen expression, without knowledge of the identity of the macaques. The cumulative scores for each animal were expressed as number of positive fields per 100 fields (\%). Selected lung sections from macaques in the control group were stained with monoclonal antibody AE1/AE3 to cytokeratin (Neomarkers) for identification of epithelial cells, according to standard immunohistochemical procedures.

SCV RT-PCR. RT-PCR with primers and probe specific for the nucleoprotein gene of SCV was used to quantify SCV in swabs, as described ${ }^{2}$. Serial dilutions of the SCV stock were used as a standard, and results were expressed as SCV equivalents per ml swab medium.

Statistical analysis. Data were compared using the Student $t$-test (antiviral activity of pegylated IFN- $\alpha$ against SCV in vitro and its biological activity in macaques) or one-way ANOVA and Dunnett's multiple-comparison test (effect of pegylated
IFN- $\alpha$ on SCV excretion, SCV replication, viral antigen expression and histological lesions in the lungs). Differences were considered significant at $P<0.05$.

\section{ACKNOWLEDGMENTS}

We thank S. Bruijns, J.M. Vrolijk, G. Aron, F. van der Panne, R. Dias d'Ullois and D. Fekkes for assistance, and J.D. Laman for advice on immunohistochemistry.

\section{COMPETING INTERESTS STATEMENT}

The authors declare that they have no competing financial interests.

Received 25 September 2003; accepted 30 January 2004

Published online at http://www.nature.com/naturemedicine/

1. Fouchier, R.A.M. et al. Koch's postulates fulfilled for SARS virus. Nature 423, 240 (2003).

2. Kuiken, T. et al. Newly discovered coronavirus as the primary cause of severe acute respiratory syndrome. Lancet 362, 263-270 (2003).

3. Marra, M.A. et al. The genome sequence of the SARS-associated coronavirus. Science 300, 1399-1404 (2003).

4. Rota, P.A. et al. Characterization of a novel coronavirus associated with severe acute respiratory syndrome. Science 300, 1394-1399 (2003).

5. Drosten, C. et al. Identification of a novel coronavirus in patients with severe acute respiratory syndrome. N. Engl. J. Med. 348, 1967-1976 (2003).

6. Ksiazek, T.G. et al. A novel coronavirus associated with severe acute respiratory syndrome. N. Engl. J. Med. 348, 1953-1966 (2003).

7. Peiris, J.S.M. et al. Coronavirus as a possible cause of severe acute respiratory syndrome. Lancet 361, 1319-1325 (2003).

8. Tsang, K.W. et al. A cluster of cases of severe acute respiratory syndrome in Hong Kong. N. Engl. J. Med. 348, 1977-1985 (2003).

9. Lee, N. et al. A major outbreak of severe acute respiratory syndrome in Hong Kong. N. Engl. J. Med. 348, 1986-1994 (2003).

10. Poutanen, S.M. et al. Identification of severe acute respiratory syndrome in Canada. N. Engl. J. Med. 348, 1995-2005 (2003).

11. Nicholls, J.M. et al. Lung pathology of fatal severe acute respiratory syndrome. Lancet $361,1773-1178(2003)$

12. Ware, L.B. \& Matthay, M.A. The acute respiratory distress syndrome. N. Engl. J. Med. 342, 1334-1349 (2000).

13. Williams, M.C. The alveolar epithelium: structure and study by immunohistochemistry. in Electron Microscopy of the Lung (ed. Schraufnagel, D.E.) 121-147 (Marcel Dekker, New York, 1990).

14. Bellum, S.C. et al. Respiratory reovirus $1 / L$ induction of intraluminal fibrosis. A model for the study of bronchiolitis obliterans organizing pneumonia. Am. J. Pathol. 150, 2243-2254 (1997)

15. London, L. et al. Respiratory reovirus $1 / \mathrm{L}$ induction of diffuse alveolar damage: pulmonary fibrosis is not modulated by corticosteroids in acute respiratory distress syndrome in mice. Clin. Immunol. 103, 284-295 (2002).

16. Pei, J., Sekellick, M.J., Marcus, P.I., Choi, I.S. \& Collisson, E.W. Chicken interferon type I inhibits infectious bronchitis virus replication and associated respiratory illness. J. Interferon Cytokine Res. 21, 1071-1077 (2001).

17. Smith, A.L., Barthold, S.W. \& Beck, D.S. Intranasally administered $\alpha / \beta$ interferon prevents extension of mouse hepatitis virus, strain JHM, into the brains of BALB/CByJ mice Ant. Res. 8, 239-245 (1987).

18. Turner, R.B., Felton, A., Kosak, K., Kelsey, D.K. \& Meschievitz, C.K. Prevention of experimental coronavirus colds with intranasal $\alpha-2 b$ interferon. J. Infect. Dis. 154, 443-447 (1986).

19. Manns, M.P. et al. Peginterferon $\alpha-2 b$ plus ribavirin compared with interferon $\alpha-2 b$ plus ribavirin for initial treatment of chronic hepatitis C: a randomised trial. Lancet $\mathbf{3 5 8}$, 958-965 (2001).

20. Cinatl, J. et al. Treatment of SARS with human interferons. Lancet 362, 293-294 (2003)

21. Bukowski, R.M. et al. Treating cancer with PEG Intron: pharmacokinetic profile and dosing guidelines for an improved interferon- $\alpha 2 b$ formulation. Cancer 95, 389-396 (2002).

22. Van Gool, A.R. et al. Serum amino acids, biopterin and neopterin during long-term immunotherapy with interferon- $\alpha$ in high-risk melanoma patients. Psychiatry Res. 119, 125-132 (2003).

23. Franks, T.J. et al. Lung pathology of severe acute respiratory syndrome (SARS): a study of 8 autopsy cases from Singapore. Hum. Pathol. 34, 743-748 (2003).

24. Bedrossian, C.W., Sussman, J., Conklin, R.H. \& Kahan, B. Azathioprine-associated interstitial pneumonitis. Am. J. Clin. Path. 82, 148-154 (1984).

25. Biron, C.A. Interferons $\alpha$ and $\beta$ as immune regulators: a new look. Immunity 14 , 661-664 (2001).

26. Finter, N.B. \& Oldham, R.K. (eds.) Interferons: In Vivo and Clinical Studies. Vol. 4 (Elsevier, Amsterdam, 1985).

27. Booth, C.M. et al. Clinical features and short-term outcomes of 144 patients with SARS in the greater Toronto area. JAMA 289, 2801-2809 (2003).

28. Peiris, J.S.M. et al. Clinical progression and viral load in a community outbreak of coronavirus-associated SARS pneumonia: a prospective study. Lancet 361, 1767-1772 (2003)

29. Fouchier, R.A. et al. Detection of influenza viruses from different species by PCR amplification of conserved sequences in the matrix gene. J. Clin. Microbiol. 38, 4096-5001 (2000) 